\title{
Simulating the Nutritional Traits of Populations at the Small Area Levels Using Spatial Microsimulation Modelling Approach
}

\author{
Md. Abdul Hakim ${ }^{1}$, Azizur Rahman ${ }^{2}$ \\ ${ }^{1}$ Department of Food Technology and Nutritional Science, Mawlana Bhashani Science and Technology University, Santosh, Bangladesh \\ ${ }^{2}$ School of Computing and Mathematics, Charles Sturt University, Wagga Wagga, Australia
}

Email address:

info.hakim.bd@gmail.com (M. A. Hakim), azrahman@csu.edu.au (A. Rahman)

\section{To cite this article:}

Md. Abdul Hakim, Azizur Rahman. Simulating the Nutritional Traits of Populations at the Small Area Levels Using Spatial Microsimulation Modelling Approach. Computational Biology and Bioinformatics. Vol. 6, No. 1, 2018, pp. 25-30. doi: 10.11648/j.cbb.20180601.13

Received: February 12, 2018; Accepted: April 3, 2018; Published: May 5, 2018

\begin{abstract}
Nutritional traits simulating at an awesome local geographic level is vital for effective nutritional promotion programs, provision of better nutritional services and population-specific nutritional planning and management. Deficient in micro-dataset readily available for attributes of individuals at small areas affects the local and national agencies on the route ahead of their smooth managing of the serious nutritional issues and related risks in the community. A solution of this ongoing challenge would be to form a method to simulate reliable small area statistics. This paper provides a dashing appraisal of the methodologies for simulating nutritional traits of populations at geographical limited areas. Findings reveal that microsimulation-based spatial models have the significant robustness over the other methods stated in this study representing a more precise means of simulatingn utrition-related traits of population at the small area levels.
\end{abstract}

Keywords: Nutritional Traits, Microsimulation Modelling, SWOT Analysis, Multilevel Models, Small Area Estimates

\section{Introduction}

Nutrition-related traits of a population in a society are significant to nutrition promotion programs and to the provision of better nutritional services [1-3]. The efforts of feasible nutritional planning generally target limited areas such as the local nutritional region or small area nutritional units, while the population-specific nutritional planning often requires precise estimates of nutritional behavior at these levels, for which nutrition-related data are not always available [4-6]. Even if regional knowledge of nutritionrelated behavior can be attained by conducting a costly sample survey, such surveys seldom generate reliable data for small geographic surroundings, i.e., Statistical Local Areas (SLAs) in Australia, countries in United States (US) or wards (electoral divisions) in United Kingdom (UK) and therefore alternative biostatical techniques are in galore need to obtain small area nutritional indicators.

The policy makers and nutritional researchers often rely on national or state-level data set to understand the nutritional needs of their communities. The lack of national dataset for traits of individuals at small area levels hampers the local and national agencies to manage the horrors of nutritional soundness and associated risk factors in the communities and so forming a model [7-9] can be a problem solving bid to simulate spatial micro-population dataset [10-12] at an awesome geographic level. These can be achieved applying Small Area Estimation (SAE) techniques, commonly known as the statistical modelling approaches, i.e., indirectstandardization and individual level modelling [1315], multilevel statistical modelling [16-20] and microsimulation modelling [21-29].

The SAE can provide robust estimates of the population nutritional behavior in small geographic areas to support comparisons within and between local areas such as SLA levels and state or national level estimates. Though the indirect standardization and individual level modelling approaches are simpler than the multilevel modelling approaches, they are not robust in terms ofc omputational processes [30-33]. The latest microsimulation modelling approaches are more advanced than the other statistical modelling methods, and they are also methodologically and 
computationally more sophisticated. However, it is yet to be assessed which small area technique produces the most valid, statistically reliable and precise estimates of nutrition-related characteristics [34-37]. The basic problem with surveys at the state or national level is that they are not designed for efficient simulation of the situation in small areas [38, 39].

Due to the lack of enough sample information in small geographic area levels, there is much possibility in using simulated or synthetic estimators for small areas [40, 41]. The estimates of small area nutrition-related traits such as the smoking behavior of youth and adults, characteristics by overweight and obesity etc. at small area levels are not readily available for policy making purposes [42-44]. This article provides a significant appraisal of the methodologies for the estimation of nutrition-related traits of populations in geographic limited areas. A wide range of methodologies have been used in simulating small area nutritional traits (Figure1). Traditionally there are two types of SAE namely direct and indirect model-based estimations. Synthetic reconstruction and reweighting are commonly used in microsimulation approaches and each is stimulated by different techniques, i.e., Combinatorial Optimization(CO), Generalized Regression Weighting (GREGWT) and Bayesian reweighting algorithms to yield simulated estimators (Figure 1). All of these SAE have not been rampantly applied for nutritional modelling, especially not for simulating nutritional traits. There are different reasons forthis, i.e., the lacuna of initial data requirements for some methods and the distribution of predictors at the smal larea levels are not known.

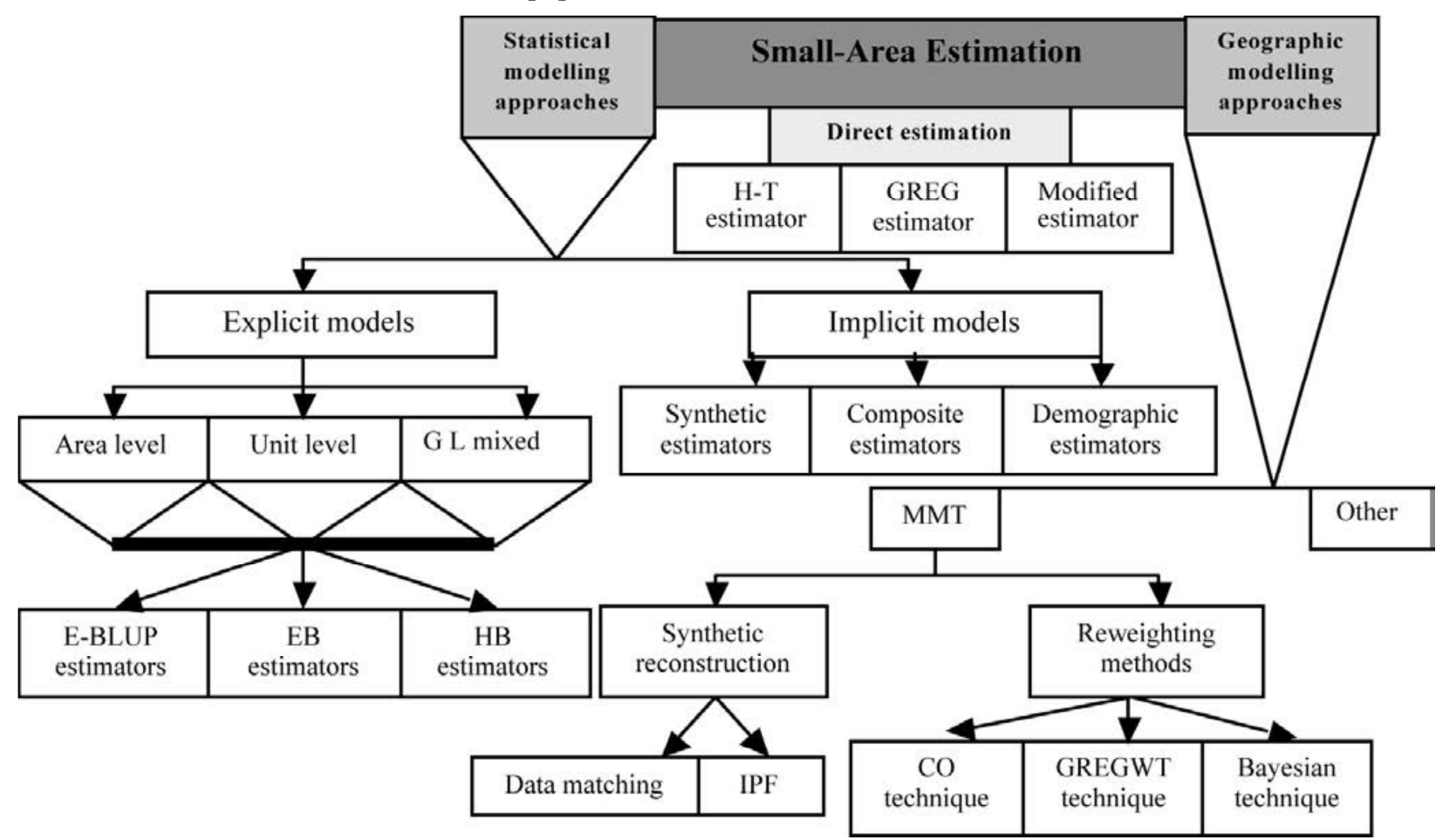

Figure 1. A summary of different techniques and estimators for small area estimation [45].

\section{Methodology and Data Sources}

This is a methodological review study to design a trendy microsimulation modelling technique for estimating nutritional characteristics of population at small area levels. A wide range of instruments have been collected from the different kinds of microsimulation methods in biostatistics, spatial approaches in nutritional physics, algorithmic gesture in computational biology, the SWOT analysis in applied sociology, Chi-square test and the GREGWT modelling in Newton-Raphson method of iteration in computational physics. These instruments have then used for examining all the statistical modelling in quest of an effective microsimulation modelling technique to simulate nutritional traits of populations at small area levels in different countries in the world.

\section{Results}

Microsimulation modelling approach is getting vast attention to the nutritional researchers for its robustness to the use in geographical information at small-area levels [46]. A growing literature indicates that microsimulation models are being popular tool in nutritional research to simulate nutrition-related behavior, future prevalence rates, cost of treatment, provision of care needs, and the potential outcomes of policy intervention at small areal evels [47-49]. This is a promising technique for creating synthetic or simulated microdata describing household characteristics at the small area level. The creation of reliable synthetic microdata at the small area level is often challenging for some regions.

Microsimulation modelling can be conducted by 
reweighting a national level sample so as to estimate the detailed socioeconomic and demographic characteristics of populations and households at the small area level. The presence of geographical information and detailed household characteristics which both have impact on nutrition-related behavior in the synthetic spatial micro-population indicates the applicability of a microsimulation modelling. The features of microsimulation modelling technology and the associated theories, tools and techniques behind this approach are provided in a number of studies and hence an appraisal of the misprovided. Two types of methodologies for creating simulated micro-population datasets are in use: i) synthetic reconstruction and ii) reweighting. The former approach includes data matching or fusion [50] and iterative proportional fitting [51, 52] while the latter utilizes GREGWT [53] and Combinatorial Optimisation (CO) [54].

GREGWT is an iterative generalise dregression algorithm written in SAS macros to calibrate survey estimates to bench marks. The use of auxiliary information improves the resulting survey estimates that a reproduced using them modified grossing factors. The algorithm used in GREGWT is based on a constrained distance function known as the truncated Chi-squared istance function that is minimised subject to the calibration equations for each small area. The method is also known as linear truncated or restricted modified Chi-square [27, 55] or the truncated linear regression method. The truncated Chi square distance function is used in the GREGWT algorithm as follows:

$$
D_{X^{2}}=\sum_{K \in S} \frac{\left(w_{K}-D_{k}\right)^{2}}{D_{k}} ; \text { for } L_{k} \leq \frac{w_{K}}{D_{k}} \leq U_{k}
$$

Where $D_{k}$ is the given sampling design weights, $W_{k}$ the new weights, and $L_{k}$ and $U_{k}$ the pre specified lower and upper bounds, respectively, for each unit $k$ in sample $S$. The basic advantage of this method over linear regression is that the new weights mustlie with inapre-specified boundary condition for each small area unit. The upper and lower limits of the boundary interval could be constant acrosss ample units or proportional to the original sampling weights. The GREGWT algorithm uses the Newton-Raphson method of iteration to minimize this distance function [56].

\section{Discussion}

Most of the developed nations are using small area estimation methodologies as an essential means to support the process of effective decision making and policy analyzing purposes for various issues at local area levels $[57,58]$. The microsimulation modelling technology-based spatial models are more precise means in which two or more sources of data can be combined $[59,60]$. The premier objective of creating simulated micro-population datasets at the small area level is to create data that doesnot currently exist for small areas and so the validation of simulated micro-data is difficult and may be considered one of the drawbacks of MMT. However, then the Bayesian prediction-based model can eclipse this drawback through simulating the complete scenario of micropopulation units at the small area level and then producing the statistical reliability measures of the SAEs from MMTbased model [61]. There are also ways to deal with the validation problem for other MMTs which are more reliable and scientifically standard means for validating the estimates from microsimulation modelling method. Yet scholars are working to improve the validation methods and/or trying to develop a further one for then on-Bayesian reweighting based microsimulation models [60]. The multilevel statistical modelling approach is frequently used in explaining the variability in human characteristics. The method has an extended ability to incorporate unexplained variability between small areas into the nutrition-related attributes estimation procedures and can be applicable to survey data that simultaneously accounts for either individual and small area level effects or small area random effects on nutritionrelated behaviors $[62,63]$. As the multilevel modelling in cludes the individual level covariates this method also imposes quitestringentdat a requirements, like individual level modelling and hence important individual level predictors of nutrition-related traits may be dropped from the model due to unknown distributions of these variables at the local level. The SAEs procedure is also rather complex in multilevel statistical modelling.

The microsimulation modelling haemerged recently as a splendid alternative for small areae stimation of nutritionrelated characteristics. The main challenge is the requirement for reliable synthetic spatial micro-data. Findings have revealed that two reweighting methods, the GREGWT and $\mathrm{CO}$ are commonly used tools to generate small area microdata. The formerutilisesatruncated Chisquared distance function and generates a set of new weights by minimising the total distance with respect to some constrain tfunctions. A comparison between the GREGWT and CO reveal that they are using quite different iterative algorithms having considerable properties. The $\mathrm{CO}$ has a tendency to include fewer households giving them higher weight and conversely the GREGWT has a tendency to select more households giving them less weight. However, the overall performances are fairly similar for both micro-data simulation techniques from the standpoint of use in microsimulation modelling. The study findings create pinch of salt on using the microsimulation modelling technology because of its containing more robustness than that of the other methods in the study on the basis of the choice of spatial scales (Figure 2). This nutritional microsimulation modelling can be an effective tool in simulating nutritional characteristics of populations at small area levels at the field of health pedagogy in nutritional biostatistics [64-68]. 


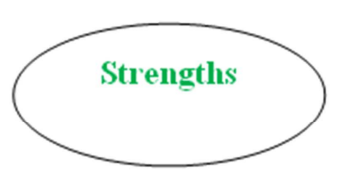

a) Sophisticated and "state of the art" method that can generate measures of statistical reliability of the estimates.

b) Robust approach in terms of the choice of further aggregation or disaggregation of the small area estimates on the basis of different spatial scales.

c) Able to utilise the small area level synthetic microdata file for further analysis and updating.

d) It is in more robustnest over the other traditional statistical approaches. a) The approach is extremely computing-intensive with regard to the size of the final file.

b) Depends solely on a good micro-data generation technique. Several techniques are in use, but a better and commonly available reweighting method is yet to be developed.

c) The resulting $\mathrm{SAE}$ estimates become statistically unreliable in case of few observation in a sample stratum.

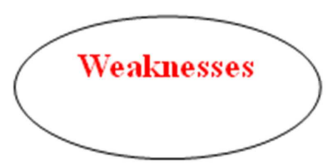

a) Expensive

b) Required skilled manpower.

a) Fruitful statistical result provider.

b) Popularizing in developing countries.

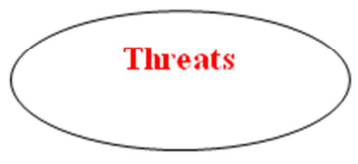

Figure 2. SWOT analysis [69-73] of spatial microsimulation modelling tetechnique.

\section{Conclusions}

This paper has reviewed the methodologies in simulating nutrition-related characteristics of populations at the small area levels. This simulation in nutritional disparities offer more informative way than that of the other approaches and it can help to direct for developing advance policies to reduce inequities across different populations. The spatial model with the static microsimulation model isused to assess small area effects of policy changes. The MMT approaches allow what-if scenarios in terms of policy changes. The MMT is a comparatively precise way to estimate small area nutritionrelated characteristics and evaluating policy changes. Future research should adopt this cozy simulating approach to get the estimates of these traits, especially the estimates of smoking behavior of adults and/or in estimating the prevalence of overweight and obesity of adults at the small area levels in Australia, the UK and the US.

\section{References}

[1] Rahman A and Chowdhury S. Determinants of chronic malnutrition among preschool children in Bangladesh. $J$ Biosoc Sci 2007; 39 (2): 161-173.

[2] Macharia PM, Ouma PO, Gogo EG, Snow RW and Noor AM. Spatial accessibility to basic public health services in South Sudan. Geospat Health 2017; 12: 106-113.

[3] Rahman A and Hakim MA. An epidemiological study on hygiene practices and malnutrition prevalence of beggars children in Bangladesh. Int J Nutr Diet 2016; 4 (1): 29-46.

[4] Seliske L, Norwood TA, McLaughlin JR, Wang S, Palleschi C and Holowaty E. Estimating micro area behavioural risk factor prevalence from large population-based surveys: a full Bayesian approach. BMC Public Health 2016; 16: 478.

[5] Bubliy OA, Loeschcke V and Imasheva AG. Genetic variation of morphological traits in Drosophila melanogaster under poor nutrition: isofemale lines and offspring-parent regression. Heredity 2001; 86: 363-369.

[6] Banerjee A and Chaudhury S. Statistics without tears: populations and samples. Ind Psychiatry J 2010; 19 (1): 60-65.

[7] Rahman A and Hakim MA. Modelling health status using the logarithmic biophysical modulator. $J$ Public Health Epidemiol 2017; 9 (5): 145-150.

[8] Hakim MA. Modelling food energy in bioenergetics. $J$ Adv Med Pharaceut Sci 2017; 14 (4): 1-7.

[9] Hakim MA. Simulating the ideal body weight in human populations. Int J Biochem Biophys 2017; 5 (4): 79-82.

[10] Rahman A and Hakim MA. Malnutrition prevalence and health practices of homeless children: a cross-sectional study in Bangladesh. Sci J Public Health 2016; 4 (1-1): 10-15.

[11] Rahman A and Hakim MA. Measuing modified mass energy equivalence in nutritional epidemiology: a proposal to adapt the biophysical modelling approach. Int J Stat Med Res 2016; 5 (3): 219-223.

[12] Rahman A. Significant risk factors for childhood malnutrition: evidence from an Asian developing country. Sci J Public Health 2016; 4 (1-1): 16-27. 
[13] Charlton J. Use of the census sample of anonymised records (SARs) and survey data in combination to obtain estimates at local authority level. Env Plan Anal 1998; 30: 775-84.

[14] Schaible W. Indirect estimators in U.S. federal programs. Springer, New York, NY, USA, 1996.

[15] Rao JNK. Small area estimation. John Wiley \& Sons, Inc., New York, NY, USA, 2003.

[16] Ghosh M and Rao JNK. Small area estimation - an appraisal. Stat Sci 1994; 9: 55-76.

[17] Rahman A, Harding A, Tanon R and Liu S. Methodological issues in spatial microsimulation modelling for small area estimation. Int J Microsimul 2010; 3 (2): 3-22.

[18] Rao JNK and Molina I. Small area estimation. John Wiley \& Sons, Inc., New York, NY, USA, 2015.

[19] Zhang X, Onufrak S, Holt JB and Croft JB. A multilevel approach for estimating small area childhood obesity prevalence at the sensus block-group level. Prev Chronic Dis 2013; 10: E68.

[20] Pfeffermann D. Small area estimation - new developments and directions. Int Stat Rev 2002; 70: 125-43.

[21] Rahman A and Harding A. Small area estimation and microsimulation modelling. Chapman and Hall/CRC, London, UK, 2016.

[22] Edwards KL and Clarke GP. The design and validation of a spatial microsimulation model of obesogenic environments for children in Leeds, UK: SimObesity. Soc Sci Med 2009; 69: 1127-34.

[23] Rahman A and Harding A. Spatial analysis of housing stress estimation in Australia with statistical validation. Aust $J$ Reg Stud 2014; 20:452-86.

[24] Rahman A and Harding A. Tenure specific small area estimation of housing stress in Australia. Paper presented at the 2013 International Indian Statistical Association (IISA) Conference in Chennai, India (January 2-5), 2013.

[25] Rahman A and Upadhyay S. A Bayesian reweighting technique for small area estimation. In: S. K. Upadhyay, U. Singh, and D. K. Dey (eds.). Current trends in bayesian methodology with applications. Chapman and Hall/CRC, London, UK, 2015; pp. 503- 19.

[26] Brown L, Yap M, Lymer S, Chin SF, Leicester S, Blake M and Harding A. Spatial microsimulation modelling of care needs, costs and capacity for self-provision: detailed regional projection for older Australians to 2020. Paper presented at the Australian Population Association Conference, Canberra, Australia, 2004.

[27] Rahman A. A review of small area estimation problems and methodological developments. University of Canberra, Canberra, Australia, 2008.

[28] Mena C, Fuentes E, Ormazabal Y and Polomo I. Geographic clustering of elderly people with above-norm anthropometric measurements and blood chemistry. Geospat Health 2017; 12: 90-95.

[29] Chin SF and Harding A. Regional dimensions: Creating synthetic small-area microdata and spatial microsimulation models. NATSEM, University of Canberra, Australia, 2006.
[30] Hakim MA. Biophysical modelling of dietary energy in biochemical modelling. Eur J Biophys 2017; 5 (3): 57-61.

[31] Bajekal M, Scholes S, Pickering K and Purdon S. Synthetic estimation of healthy lifestyles indicators: stage 1 report. National Centre for Social Research, London, UK, 2004.

[32] Rahman A, Hakim MA, Hanif MA, Islam MR and Kamruzzaman M. Dietary practices, health status and hygiene observance of slum kids: a pilot study in an Asian developing country. JP J Biostat 2016; 13 (2): 195-208.

[33] Rahman A. Small area housing stress estimation in Australia: calculating confidence intervals for a spatial microsimulation model. Commun Stat 2017; 84 (9):1-19.

[34] Hakim MA. Mathematical modelling of energy balancing for diet plannning in nutritional physics. Int J Nutr Diet 2017; 5 (1): 29-41.

[35] Rahman A and Hasan N. Modelling effects of KM and HRM processes to the organizational performance and employee's job satisfaction. Int J Business Manage 2017; 12 (7): 35.

[36] Hakim MA and Rahman A. Health and nutritional condition of street children of Dhaka city: an empirical study in Bangladesh. Sci J Public Health 2016; 4(1-1): 6-9.

[37] Islam J, Rahman A and Boland G. Nexus of learning style with satisfaction and success of accounting students: a cultural study at an Australian university. Int J Learn Change 2011; 5 (3-4): 288-304.

[38] Rahman A. Small area estimation through spatial microsimulation models: Some methodological issues. Paper presented at the 2nd General Conference of the International Microsimulation Association in Ottawa, Canada, 2009; p. 145 (June 8-10).

[39] Heady P, Clarke P, Brown G, Ellis K, Heasman D, Hennell S, Longhurst $\mathrm{J}$ and Mitchell B. Model-based small area estimation series no. 2: small area estimation project report. Office for National Statistics, London, UK, 2003.

[40] Rahman A and Harding A. Social and health costs of tobacco smoking in Australia: level, trend and determinants. Int J Stat Syst 2011; 6:375-87.

[41] Kamruzzaman, Hakim MA, Hanif MA, Rahman MH, Islam MA, Talukder MJ and Islam MR. Patterns of behavioral changes among adolescent smokers: an empirical study. Front Biomed Sci 2016; 1 (1): 1-6.

[42] Kamruzzaman M and Hakim MA. Basic rights on the wane, human rights on brown study: a case study on thrown away children in Bangladesh. Int J Environ Plan Manage 2016; 2 (4): 29-35.

[43] Hakim MA and Kamruzzaman M. The dance of poverty and education for childhood nutritional victimization in Bangladesh. J Biol Environ Eng 2016; 1 (1): 6-9.

[44] Hakim MA, Talukder MJ and Islam MS. Nutritional status and hygiene behavior of government primary school kids in central Bangladesh. Sci J Public Health 2015; 3(5): 638-642.

[45] Rahman A. Estimating small area health-related characteristics of populations: a methodological review. Geospat Health 2017; 12: 3-14.

[46] Burden S and Steel D. Constraint choice for spatial microsimulation. Popul Space Place 2016; 22:568-83. 
[47] Brown L and Harding A. The new frontier of health and aged care: using microsimulation to assess policy options: tools for microeconomic policy analysis. Productivity Commission of Australia, Canberra, Australia, 2005.

[48] Hakim MA. Biophysical modelling of cellular energy in human dietetics: an appraisal in nutritional physics and cell biology. Am J Food Sci Nutr Res 2017; 4 (4): 125-129.

[49] Procter KL, Clarke GP, Ransley JK and Cade J. Micro-level analysis of childhood obesity, diet, physical activity, residential socio-economic and social capital variables: where are the obesogenic environments in Leeds? Area 2008; 40:323-40.

[50] Tranmer M, Pickles A, Fieldhouse E, Elliot M, Dale A, Brown M, Martin D, Steel D and Gardiner C. Microdata for small areas. The Cathie Marsh Centre for Census and Survey Research (CCSR), University of Manchester, Manchester, UK, 2001.

[51] Williamson P. Community health care policies for the elderly: a microsimulation approach. School of Geography, University of Leeds, Leeds, UK, 1992.

[52] Norman P. Putting iterative proportional fitting on the researcher's desk. School of Geography, University of Leeds, Leeds, UK, 1999.

[53] Bell P, 2000. GREGWT and TABLE macros user guide (unpublished). Australian Bureau of Statistics, Canberra, Australia, 2000.

[54] Huang Z and Williamson P. A comparison of synthetic reconstruction and combinatorial optimisation approaches to the creation of small-area microdata. Population Microdata Unit, Department of Geography, University of Liverpool, Liverpool, UK, 2001.

[55] Singh AC and Mohl CA. Understanding calibration estimators in survey sampling. Survey Methodol 1996; 22:107-15.

[56] http://mathworld. wolfram.com/NewtonsMethod.html.

[57] Rahman A, Chowdhury S and Hossain D. Acute malnutrition in Bangladeshi children: levels and determinants. Asia Pac J Public Health 2009; 21 (1): 294-302.

[58] Hakim MA. Simulating the ideal body mass in adult human samples. Int J Sport Sci Phys Educ 2017; 2 (4): 57-60.

[59] Rahman A, Harding A, Tanton R and Liu S. Simulating the characteristics of populations at the small area level: New validation techniques for a spatial microsimulation model in Australia. Comput Stat Data Analysis 2013; 57 (1): 149-165.

[60] Whitworth A, Carter E, Ballas D and Moon G. Estimating uncertainty in spatial microsimulation approaches to small area estimation: A new approach to solving an old problem. Comput Environ Urban Syst 2017; 63: 50-57.

[61] Tanon R, Vidyattama Y, Nepal B and McNamara J. Small area estimation using a reweighting algorithm. J Royal Stat Society 2011; 174 (4): 931-951.

[62] Hakim MA and Kamruzzaman M. Nutritional status of preschoolers in four selected fisher communities. Am J Life Sci 2015; 3(4): 332-336.

[63] Sakboonyarat B, Chokcharoensap K, Meesaeng M, Jaisue N, Janthayanont D and Srisawat P. Prevalence and associated factors of Low Back Pain (LBP) among adolescents in Central, Thailand. Glob J Health Sci 2018; 10 (2): 49-59.

[64] Hakim MA. Malnutrition prevalence and nutrition counseling in developing countries: A case study. Int $J$ Nurs Health Sci 2016; 3(3):19-22.

[65] [65] Kuddus A, Rahman A, Talukder MR and Hoque A. A modified SIR model to study on physical behaviour among smallpox infective population in Bangladesh. Am J Math Stat 2014; 4(5): 231-239.

[66] Kamruzzaman M and Hakim MA. Socio-economic status of child beggars in Dhaka City. J Soc Sci Human 2015; 1(5): 516-520.

[67] Rahman A, Gao J and Ahmed S. An assessment of the effects of prior distribution on the Bayesian predictive inference. Int $J$ Stat Prob 2016; 5 (5): 31-42.

[68] Poole SA, Hart CN, Jelalian E and Raynor HA. Relationship between dietary energy and dietary quality in overweight young children: A cross-sectional analysis. Pediatr Obesity 2016; 11(2):128-135.

[69] Armstrong M. A handbook of human resource management practice $\left(10^{\text {th }}\right.$ ed). Kogan Page, London, 2006.

[70] Osita C, Onyebuchi I and Justina N. Organization's stability and productivity: the role of SWOT analysis. Int J Innov Appl Res 2014; 2 (9): 23-32.

[71] Birkenmaier J. The practice of generalist social work. New York, NY: Routledge, 2001.

[72] Kamruzzaman M and Hakim MA. A review on child labour criticism in Bangladesh: an analysis. Int J Sport Sci Phys Educ 2018; 3 (1): 1-8.

[73] Linkon MR, Prodhan UK et al. Comperative analysis of the physico-chemical and antioxidant properties of honey available in Tangail, Bangladesh. Int J Res Eng Technol 2015; 4 (3): 89-92. 